\title{
A NOTE ON DISCRETE SOLUTIONS \\ OF THE PLATEAU PROBLEM
}

\author{
TAKUYA TSUCHIYA
}

\begin{abstract}
In this paper we prove theorems for convergence of discrete solutions of the Plateau problem under the assumption that the contour is rectifiable.
\end{abstract}

\section{INTRODUCTION}

In [7] the discrete solutions of the Plateau problem were defined, and some theorems for its convergence were proved under a very restrictive condition. The purpose of this paper is to show that we can obtain the same conclusions if the contour is rectifiable.

It is well known [2, pp. 107-118] that the Plateau problem can be defined as the following variational problem:

Let $D=\left\{(u, v) \in \mathbf{R}^{2} \mid u^{2}+v^{2}<1\right\}$ be the unit disk with boundary $\partial D$ and let $\Gamma$ be a Jordan curve in $n$-dimensional Euclidean space $\mathbf{R}^{n}, n \geq 2$. Let $C\left(\bar{D} ; \mathbf{R}^{n}\right)$ be the space of continuous maps from $\bar{D}$ into $\mathbf{R}^{n}$, and let $H^{1}\left(D ; \mathbf{R}^{n}\right)$ be the ordinary Sobolev space (for the exact definitions, see [7]). We define the class of maps by

$$
X_{\Gamma}=\left\{f \in C\left(\bar{D} ; \mathbf{R}^{n}\right) \cap H^{1}\left(D ; \mathbf{R}^{n}\right)|f(\partial D)=\Gamma, f|_{\partial D} \text { is monotone }\right\},
$$

where monotone means that, for each $p \in \Gamma,\left(\left.f\right|_{\partial D}\right)^{-1}(p) \subset \partial D$ is connected. $X_{\Gamma}$ may be empty [4, p. 58], but if $\Gamma$ is rectifiable, then $X_{\Gamma} \neq \varnothing[2$, pp. 129131]. We choose six arbitrary distinct points $z_{1}, z_{2}, z_{3} \in \partial D$ and $\zeta_{1}, \zeta_{2}, \zeta_{3} \in$ $\Gamma$, and we define the subset of $X_{\Gamma}$ by

$$
X_{\Gamma}^{\mathrm{tp}}=\left\{f \in X_{\Gamma} \mid f\left(z_{i}\right)=\zeta_{i}, \quad i=1,2,3\right\},
$$

where the superscript "tp" stands for "three-point condition". The Plateau problem is to find stationary points of the energy functional

$$
E(f)=\frac{1}{2} \iint_{D}\left(\left|f_{u}\right|^{2}+\left|\hat{f}_{v}\right|^{2}\right) d u d v
$$

in $X_{\Gamma}^{\mathrm{tp}}$, where $f_{u}=\left(\partial f_{1} / \partial u, \ldots, \partial f_{n} / \partial u\right)$ and $f_{v}=\left(\partial f_{1} / \partial v, \ldots, \partial f_{n} / \partial v\right)$. The notation $|\cdot|$ means Euclidean norm.

A solution of the Plateau problem is called a minimal surface spanned in $\Gamma$ even if it is not a minimal point of the energy functional. For the existence of

Received March 30, 1987; revised February 1, 1989.

1980 Mathematics Subject Classification (1985 Revision). Primary 49F10, 65E05, 65N30. 
the minimal surfaces the following theorem is known [2, pp. 101-105; 4, p. 71]: Theorem A (Douglas-Rado). Let $e_{\Gamma}=\inf \left\{E(f): f \in X_{\Gamma}^{\mathrm{tp}}\right\}$. If $X_{\Gamma}^{\mathrm{tp}} \neq \varnothing$, then there exists a map $x \in X_{\Gamma}^{\mathrm{tp}}$ such that $E(x)=e_{\Gamma}$.

An $x$ as in Theorem A is called a Douglas solution. Evidently, a Douglas solution is a minimal surface.

In $\S 2$ we define a (stable) discrete minimal surface using the simplest finite element scheme. In $\S 3$ we prove the relative compactness of bounded subsets of discrete maps when the Jordan curve is rectifiable. In [7] a very restrictive condition was assumed to prove the relative compactness, so $\S 3$ is the main part of this paper.

\section{Definition of THE DisCRETE Minimal SURFACE}

Let $\Omega \subset D$ be a regular triangulation of $D$ with $\bar{\Omega}=\bigcup K_{i}$, where $K_{i}$ are triangles. With the triangulation $\Omega$ we associate the mesh size of $\Omega$ defined by

$$
|\Omega|=\max _{i} \operatorname{diam}\left(K_{i}\right) .
$$

We assume that there exists a positive constant $\omega$ which is independent of the triangulation $\Omega$ such that the following inequality holds for each triangle $K_{i} \subset \Omega$ :

$$
\operatorname{diam}\left(K_{i}\right) / \rho\left(K_{i}\right) \leq \omega,
$$

where $\rho\left(K_{i}\right)=\sup \left\{\operatorname{diam}(S) ; K_{i} \supset S:\right.$ ball $\}$.

Let $S_{\Omega}$ be the set of functions which are continuous on $\bar{\Omega}$ and linear on each triangle $K_{i}$. Let $\mathbf{S}_{\Omega}$ be the set of maps from $\bar{\Omega}$ into $\mathbf{R}^{n}$ such that each component function belongs to $S_{\Omega}$. Let $N_{\Omega}=\left\{b_{i}\right\}_{i=1}^{N+N^{\prime}}$ be the set of nodal points of $\Omega$ where $b_{i} \in \Omega^{\circ}$, the interior of $\Omega$, for $1 \leq i \leq N$, and $b_{i} \in \partial \Omega$ for $N+1 \leq i \leq N+N^{\prime}$. We number $\left\{b_{N+1}, \ldots, b_{N+N^{\prime}}\right\}=N_{\Omega} \cap \partial D$ in counter-clockwise order. We assume that

$$
\Omega \text { is of nonnegative type. }
$$

For the definition of the term "nonnegative type", see $[1,7]$. This assumption is for the discrete maximum principle [7, Lemma 3]. We introduce the admissible class of triangulations of $D$ defined by

$$
\Delta^{\mathrm{tp}}=\left\{\Omega \mid z_{1}, z_{2}, z_{3} \in N_{\Omega}, \Omega \text { satisfies }(\mathrm{H} 1),(\mathrm{H} 2)\right\} .
$$

When $\Omega$ is given, we define

$$
X_{\Gamma, \Omega}=\left\{f \in \mathbf{S}_{\Omega}\left|f\left(N_{\Omega} \cap \partial D\right) \subset \Gamma, f\right|_{\partial D} \text { is } d \text {-monotone }\right\},
$$

where $d$-monotone means that the order of nodal points on $\Gamma$ is the same as the order of nodal points on $\partial D$. Let

$$
X_{\Gamma, \Omega}^{\mathrm{tp}}=\left\{f \in X_{\Gamma, \Omega} \mid f\left(z_{i}\right)=\zeta_{i}, \quad i=1,2,3\right\},
$$

and let $E_{\Omega}(f)$ be the energy functional on $\Omega$ defined by

$$
E_{\Omega}(f)=\frac{1}{2} \iint_{\Omega}\left(\left|f_{u}\right|^{2}+\left|f_{v}\right|^{2}\right) d u d v
$$


We extend $f \in \mathbf{S}_{\Omega}$ to $D-\Omega$ as follows:

If $p \in \partial \Omega$ and $p \notin N_{\Omega}$, there exists an exterior normal half-line $L_{p}$ of $\partial \Omega$ on $p$. For arbitrary $q \in L_{p} \cap(D-\Omega)$, we define $f(q)=f(p)$. Then the following estimate is valid:

$$
E_{\Omega}(f) \leq E(f) \leq(1+C|\Omega|) E_{\Omega}(f) \text { for any } f \in \mathbf{S}_{\Omega},
$$

where $C$ is a constant which is independent of $\Omega$ and $f$.

Definition 1. Let $\Omega \in \Delta^{\mathrm{tp}}$.

(D1) $f \in X_{\Gamma, \Omega}^{\mathrm{tp}}$ is a stable $d$-minimal surface if there exists a positive constant $\delta$ such that $\|f-g\|_{C\left(\bar{\Omega} ; \mathbf{R}^{n}\right)}<\delta$ implies $E_{\Omega}(f) \leq E_{\Omega}(g)$ for $g \in X_{\Gamma, \Omega}^{\mathrm{tp}}$.

(D2) $f \in X_{\Gamma, \Omega}^{\mathrm{tp}}$ is the $d$-Douglas solution if $E_{\Omega}(f)=\inf \left\{E_{\Omega}(g): g \in X_{\Gamma, \Omega}^{\mathrm{tp}}\right\}$.

\section{Relative compactNesS}

First, we recall a useful lemma [2, pp. 101-102; 4, pp. 67-68]. For any $z \in \mathbf{R}^{2}$ and any $r>0$ we define

$$
C_{r, z}=\bar{D} \cap\left\{w \in \mathbf{R}^{2}:|w-z|=r\right\} .
$$

For $f \in X_{\Gamma, \Omega}^{\mathrm{tp}}$ we denote by $l\left(f, C_{r, z}\right)$ the length of the image $f\left(C_{r, z}\right)$. Let $M$ be a constant with $e_{\Gamma}<M$.

Lemma 2. For arbitrary $\delta, 0<\delta<1$, and $f \in X_{\Gamma, \Omega}^{\mathrm{tp}}$ with $E(f) \leq M$, there exists $\rho, \delta \leq \rho \leq \delta^{1 / 2}$, depending on $f$ and $z$ such that

$$
l\left(f, C_{\rho, z}\right)^{2} \leq \lambda(\delta),
$$

where $\lambda(\delta)=8 \pi M / \log (1 / \delta)$.

For $\Omega \in \Delta^{\mathrm{tp}}$ and $f \in X_{\Gamma, \Omega}^{\mathrm{tp}}$ we define

$$
L(\Omega, f)=\max \left\{\left|f\left(b_{i}\right)-f\left(b_{i+1}\right)\right|: b_{i} \in N_{\Omega} \cap \partial D, \quad i=N+1, \ldots, N+N^{\prime}\right\},
$$

where $b_{N+N^{\prime}+1}=b_{N+1}$. The following lemma is valid.

Lemma 3. Let $\Delta^{\mathrm{tp}} \supset\left\{\Omega_{n}\right\}_{n=1}^{\infty}$ be such that $\lim _{n \rightarrow \infty}\left|\Omega_{n}\right|=0$, and let $f_{n} \in$ $X_{\Gamma, \Omega_{n}}^{\mathrm{tp}}$. Suppose that $\Gamma$ is rectifiable and $E\left(f_{n}\right) \leq M$ for any $n$. Then $\lim _{n \rightarrow \infty} L\left(\Omega_{n}, f_{n}\right)=0$.

Proof. The proof is by contradiction. Assume that $\limsup _{n \rightarrow \infty} L\left(\Omega_{n}, f_{n}\right)>0$. Then there exists a positive constant $\varepsilon_{0}$ such that, for any $\xi>0$, there exist a positive integer $m$ and $b_{i} \in N_{\Omega_{m}} \cap \partial D$ such that

$$
\left|\Omega_{m}\right|<\xi \text { and }\left|f_{m}\left(b_{i}\right)-f_{m}\left(b_{i+1}\right)\right| \geq \varepsilon_{0} .
$$

For $b_{i} \in N_{\Omega_{m}} \cap \partial D$ and $f_{m} \in X_{\Gamma, \Omega_{m}}^{\mathrm{tp}}$ as in (3.2), a pair $\left(\alpha_{1}, \alpha_{2}\right) \quad\left(\alpha_{i} \in\right.$ $\left.f_{m}\left(N_{\Omega_{m}} \cap \partial D\right), i=1,2\right)$ is said to be admissible if it satisfies the following properties: $\Gamma_{1}$, one of the two connected components of $\Gamma-\left\{\alpha_{1}, \alpha_{2}\right\}$, contains at least two of $\left\{\zeta_{1}, \zeta_{2}, \zeta_{3}\right\}$, and the other connected component $\Gamma_{2}$ contains 
$f_{m}\left(b_{i}\right)$ and $f_{m}\left(b_{i+1}\right)$. If $\left\{\zeta_{1}, \zeta_{2}, \zeta_{3}\right\} \cap\left\{f_{m}\left(b_{i}\right), f_{m}\left(b_{i+1}\right)\right\} \neq \varnothing$, for example in the case of $\zeta_{1}=f_{m}\left(b_{i}\right)$, a pair $\left(\alpha_{1}, \alpha_{2}\right)$ such that $\alpha_{1}=\zeta_{1}=f_{m}\left(b_{i}\right)$ and $\Gamma_{1}$ contains at least one of $\left\{\zeta_{1}, \zeta_{2}, \zeta_{3}\right\}$ and $\Gamma_{2}$ contains $f_{m}\left(b_{i+1}\right)$ is also said to be admissible.

By a topological argument we can show that there exists a positive constant $\eta$ depending on $\left\{\Gamma, \zeta_{1}, \zeta_{2}, \zeta_{3}\right\}$ and $\varepsilon_{0}$ such that $\left|\alpha_{1}-\alpha_{2}\right| \geq \eta$ for any admissible pairs $\left(\alpha_{1}, \alpha_{2}\right)$ on $\Gamma$.

Let $b_{k}, b_{h} \in N_{\Omega_{m}} \cap \partial D$ be such that all of $\left(f_{m}\left(b_{k+p}\right), f_{m}\left(b_{h+q}\right)\right) \quad(p, q=$ $0,1)$ are admissible pairs. For $b_{j} \in N_{\Omega_{m}} \cap \partial D$, we denote by $\operatorname{seg}\left(b_{j}\right)$ the segment which connects $f_{m}\left(b_{j}\right)$ and $f_{m}\left(b_{j+1}\right)$.

Lemma 4. Assume that there exist $\beta_{1} \in \operatorname{seg}\left(b_{k}\right)$ and $\beta_{2} \in \operatorname{seg}\left(b_{h}\right)$ such that $\left|\beta_{1}-\beta_{2}\right|<\eta / 2$. Then we have

$$
\left|f_{m}\left(b_{k}\right)-f_{m}\left(b_{k+1}\right)\right|+\left|f_{m}\left(b_{h}\right)-f_{m}\left(b_{h+1}\right)\right|>\eta .
$$

Proof. Since $\left|f_{m}\left(b_{k+p}\right)-f_{m}\left(b_{h+q}\right)\right| \geq \eta \quad(p, q=0,1)$, we obtain (3.3) easily.

Let $l(\Gamma)$ be the length of $\Gamma$. Let $A$ be the least integer that satisfies

$$
\frac{l(\Gamma)-\varepsilon_{0}}{\eta} \leq A
$$

We take sufficiently small $\delta, 0<\delta<1$, such that

$$
\begin{gathered}
(2 A-1)(\lambda(\delta))^{1 / 2}<\eta / 2, \\
2\left(A \delta^{1 / 2}+\gamma(\delta)\right)<\min \left\{\left|z_{i}-z_{j}\right|: i \neq j\right\},
\end{gathered}
$$

where $\gamma(\delta)=\delta^{2^{4-1}}$. We set $\xi=\gamma(\delta)$ in (3.2), and we choose and fix a positive integer $m$ and $b_{i} \in N_{\Omega_{m}} \cap \partial D$ as in (3.2). Then we have

$$
\left|\Omega_{m}\right|<\delta^{2^{4-1}} .
$$

Let $z \in \partial D$ be the center of the shorter arc $\widehat{b}_{i} b_{i+1}$. By Lemma 2 there exists a positive constant $\rho, \delta \leq \rho \leq \delta^{1 / 2}$, such that $l\left(f_{m}, C_{\rho, z}\right) \leq \lambda(\delta)^{1 / 2}$. Let $l_{1}$ and $r_{1}$ be the left and right endpoint of $C_{\rho, z}$ on $\partial D$, respectively. Suppose that $l_{1} \in{\widehat{b_{k_{1}} b}}_{k_{1}+1}$ and $r_{1} \in{\widehat{b_{h_{1}} b}}_{h_{1}+1}$, where $b_{k_{1}}, b_{h_{1}} \in N_{\Omega_{m}} \cap \partial D$. Note that the pair $\left(f_{m}\left(b_{k_{1}}\right), f_{m}\left(b_{h_{1}}\right)\right)$ is not admissible in the extraordinary case like Figure 1 .

However, in such a case we can obtain a contradiction and prove this lemma immediately. Hence we may assume without loss of generality that all of the pairs $\left(f_{m}\left(b_{k_{1}+p}\right), f_{m}\left(b_{h_{1}+q}\right)\right) \quad(p, q=0,1)$ are admissible because of (3.6). Note that, by (3.7), $b_{k_{1}}, b_{h_{1}}$ and $b_{i}$ are distinct. From (3.1) and (3.5) we have 

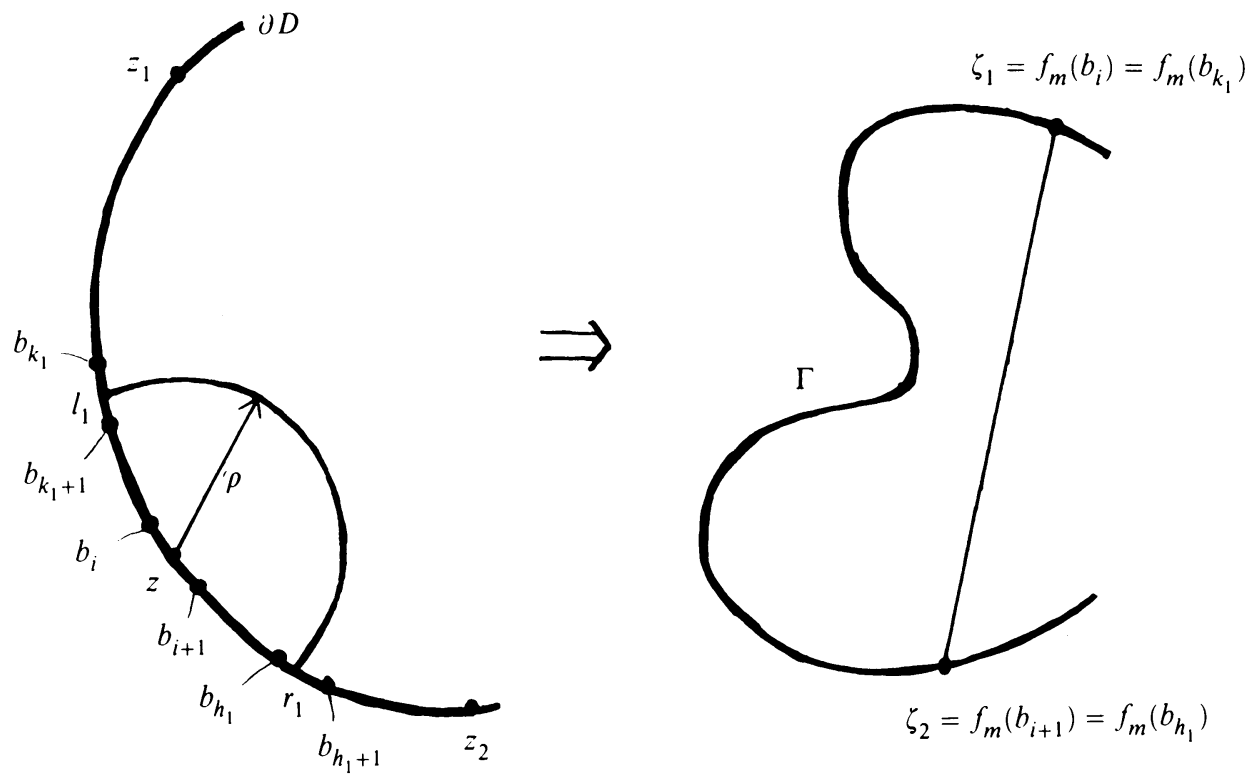

Figure 1

$\left|f_{m}\left(l_{1}\right)-f_{m}\left(r_{1}\right)\right|<(\eta / 2) /(2 A-1) \leq \eta / 2$. Thus, from Lemma 4, we obtain

$$
\left|f_{m}\left(b_{k_{1}}\right)-f_{m}\left(b_{k_{1}+1}\right)\right|+\left|f_{m}\left(b_{h_{1}}\right)-f_{m}\left(b_{h_{1}+1}\right)\right|>\eta .
$$

By Lemma 2 there exist positive constants $\theta_{1}, \rho^{2} \leq \theta_{1} \leq \rho$, and $\mu_{1}$, $\rho^{2} \leq \mu_{1} \leq \rho \quad\left(\delta^{2} \leq \theta_{1}, \mu_{1} \leq \delta^{1 / 2}\right)$, such that

$$
l\left(f_{m}, C_{\theta_{1}, l_{1}}\right)<\lambda\left(\rho^{2}\right)^{1 / 2} \leq \lambda(\delta)^{1 / 2}<\frac{\eta}{2(2 A-1)}, \quad l\left(f_{m}, C_{\mu_{1}, r_{1}}\right)<\frac{\eta}{2(2 A-1)} .
$$

Let $l_{2}$ be the left endpoint of $C_{\theta_{1}, l_{1}}$ and $r_{2}$ the right endpoint of $C_{\mu_{1}, r_{1}}$. Let $b_{k_{2}}, b_{h_{2}} \in N_{\Omega_{m}} \cap \partial D$ be nodal points such that $l_{2}$ and $r_{2}$ are on the arcs $\widehat{b_{k_{2}} b}{ }_{k_{2}+1}$ and ${\widehat{b_{h_{2}} b}}_{h_{2}+1}$, respectively. Again, we may assume that all pairs $\left(f_{m}\left(b_{k_{2}+p}\right)\right.$, $\left.f_{m}\left(b_{h_{2}+q}\right)\right)^{2}(p, q=0,1)$ are admissible because of (3.6). By (3.7), $b_{k_{1}}, b_{h_{j}}$ $(j=1,2)$ and $b_{i}$ are distinct. From (3.1) and (3.5) we have $\left|f_{m}\left(l_{2}\right)-f_{m}\left(r_{2}\right)\right|<$ $3(\eta / 2) /(2 A-1) \leq \eta / 2$. Thus, by Lemma 4 and (3.8), we obtain

$$
\sum_{j=1}^{2}\left(\left|f_{m}\left(b_{k_{j}}\right)-f_{m}\left(b_{k_{j}+1}\right)\right|+\left|f_{m}\left(b_{h_{j}}\right)-f_{m}\left(b_{h_{j}+1}\right)\right|\right)>2 \eta .
$$

Repeating this procedure $A$ times, we conclude that there exist $2 A$ distinct nodal points on $\partial D$ such that

$$
\sum_{j=1}^{A}\left(\left|f_{m}\left(b_{k_{j}}\right)-f_{m}\left(b_{k_{,}+1}\right)\right|+\left|f_{m}\left(b_{h_{j}}\right)-f_{m}\left(b_{h_{j}+1}\right)\right|\right)>A \eta .
$$


By (3.4) the right side of (3.9) is greater than $l(\Gamma)-\varepsilon_{0}$. Thus we obtain

$$
\begin{aligned}
l(\Gamma) & \geq \sum_{i=N+1}^{N+N^{\prime}}\left|f_{m}\left(b_{i}\right)-f_{m}\left(b_{i+1}\right)\right| \\
& \geq \sum_{j=1}^{A}\left(\left|f_{m}\left(b_{k_{j}}\right)-f_{m}\left(b_{k_{j}+1}\right)\right|+\left|f_{m}\left(b_{h_{j}}\right)-f_{m}\left(b_{h_{j}+1}\right)\right|\right)+\varepsilon_{0}>l(\Gamma) .
\end{aligned}
$$

This is a contradiction, hence Lemma 3 is proved.

Corollary 5. Let $\Delta^{\mathrm{tp}} \supset\left\{\Omega_{n}\right\}_{n=1}^{\infty}$ be such that $\lim _{n \rightarrow \infty}\left|\Omega_{n}\right|=0$, and let $f_{n} \in$ $X_{\Gamma, \Omega_{n}}^{\mathrm{tp}}$. Suppose that $\Gamma$ is rectifiable and $E\left(f_{n}\right) \leq M$ for any $n$. Then, for any $\varepsilon>0$, there exist $\delta>0$ and positive integer $n_{1}$ such that

$$
|s-t|<\delta \text { implies }\left|f_{n}(s)-f_{n}(t)\right|<\varepsilon,
$$

for any $s, t \in \partial D$ and $n \geq n_{1}$.

Proof. By a topological argument we can show that, for any $\varepsilon$ with $0<\varepsilon<$ $\min \left\{\left|\zeta_{i}-\zeta_{j}\right|: i \neq j\right\}$, there exists $\tau>0$ such that, if $\left|\alpha_{1}-\alpha_{2}\right|<\tau, \alpha_{1}, \alpha_{2} \in \Gamma$, then the diameter of the smaller connected component of $\Gamma-\left\{\alpha_{1}, \alpha_{2}\right\}$ is less than $\varepsilon$.

Suppose that $\varepsilon>0$ is given and $\tau>0$ is chosen in the above manner. By Lemma 3 there exists a positive integer $n_{1}$ such that $L\left(\Omega_{n}, f_{n}\right)<\tau / 3$ for all $n \geq n_{1}$. We choose $\delta>0$ such that $\lambda(\delta)^{1 / 2}<\tau / 3$ and $2 \delta^{1 / 2}<$ $\min \left\{\left|z_{i}-\bar{z}_{j}\right|: i \neq j\right\}$. By Lemma 2 , for any $s \in \partial D$, there exists $\rho, \delta \leq \rho \leq$ $\delta^{1 / 2}$, depending on $s, \delta$ and $f_{n}$, such that $l\left(f_{n}, C_{\rho, s}\right)<\tau / 3$. Let $l, r \in \partial D$ be the left and right endpoints of $C_{\rho, s}$, and let $b_{i}, b_{j} \in N_{\Omega_{n}} \cap \partial D$ be such that $l$ and $r$ are on the arcs ${\widehat{b_{i} b}}_{i+1}$ and ${\widehat{b_{j-1}}}_{j}$, respectively. Since $L\left(\Omega_{n}, f_{n}\right)<\tau / 3$, we obtain

$$
\left|f_{n}\left(b_{i}\right)-f_{n}\left(b_{j}\right)\right| \leq\left|f_{n}\left(b_{i}\right)-f_{n}(l)\right|+\left|f_{n}(l)-f_{n}(r)\right|+\left|f_{n}(r)-f_{n}\left(b_{j}\right)\right|<\tau .
$$

Thus we conclude that the diameter of $\Gamma_{1}$, the smaller connected component of $\Gamma-\left\{f_{n}\left(b_{i}\right), f_{n}\left(b_{j}\right)\right\}$, is less than $\varepsilon$, and, for any $t \in \partial D$ with $|s-t|<\delta, f_{n}(s)$ and $f_{n}(t)$ are in the convex hull of $\Gamma_{1}$. Hence we obtain $\left|f_{n}(s)-f_{n}(t)\right|<\varepsilon$.

Lemma 6. Let $\Delta^{\mathrm{tp}} \supset\left\{\Omega_{n}\right\}_{n=1}^{\infty}$ be such that $\lim _{n \rightarrow \infty}\left|\Omega_{n}\right|=0$, and let $f_{n} \in$ $X_{\Gamma, \Omega_{n}}^{\mathrm{tp}}$. Suppose that $\Gamma$ is rectifiable and $E\left(f_{n}\right)$ are uniformly bounded. Then there exists a subsequence $\left\{f_{n_{i}}\right\}$ such that $\left.f_{n_{i}}\right|_{\partial D}$ converges uniformly to a continuous map $\varphi \in C(\partial D)$ on $\partial D$. Moreover, $\varphi(\partial D)=\Gamma$ and $\varphi$ is monotone. Proof. The proof is similar to that of the Ascoli-Arzelà theorem. Let $\psi_{n}=$ $\{(\cos (2 \pi i / n), \sin (2 \pi i / n)): i=0, \ldots, n-1\}$, and let $\Psi=\bigcup_{n=1}^{\infty} \psi_{n}$. Since $\Psi$ is countable, we can number $\Psi$ as $\Psi=\left\{\gamma_{1}, \gamma_{2}, \ldots\right\}$. By the diagonal method we choose a subsequence $\left\{f_{n_{i}}\right\}$ such that, for each $j, f_{n_{i}}\left(\gamma_{j}\right)$ converges as $n_{i} \rightarrow \infty$.

Suppose that an arbitrary $\varepsilon>0$ is given. For this $\varepsilon$ we choose $\delta>0$ and a positive integer $n_{1}$ as in Corollary 5 . Let $K$ be a positive integer such that 
the length of an edge of the regular $K$-gon inscribed $\partial D$ is less than $\delta$, that is, $2 \sin (\pi / K)<\delta$. Let $\psi_{K}=\left\{\xi_{1}, \ldots, \xi_{K}\right\}$, and let $n_{2}$ be a positive integer such that $\left|f_{n_{i}}\left(\xi_{k}\right)-f_{n_{j}}\left(\xi_{k}\right)\right|<\varepsilon$, for $n_{i}, n_{j} \geq n_{2}$ and $k=1, \ldots, K$. For arbitrary $s \in \partial D$ there exists $\xi_{k} \in \psi_{K}$ such that $\left|s-\xi_{k}\right|<\delta$. Thus, by Corollary 5, we obtain

$$
\left|f_{n_{i}}(s)-f_{n_{j}}(s)\right| \leq\left|f_{n_{i}}(s)-f_{n_{i}}\left(\xi_{k}\right)\right|+\left|f_{n_{i}}\left(\xi_{k}\right)-f_{n_{j}}\left(\xi_{k}\right)\right|+\left|f_{n_{j}}\left(\xi_{k}\right)-f_{n_{j}}(s)\right|<3 \varepsilon,
$$

for $n_{i}, n_{j} \geq n_{0}=\max \left\{n_{1}, n_{2}\right\}$. Since $n_{0}$ is independent of $s,\left\{f_{n_{i}}\right\}$ converges uniformly on $\partial D$. The last part of the lemma is obvious.

\section{ThEOREMS}

Using Lemma 6, we obtain the following theorems. The proofs of the theorems are quite similar to those of the theorems in [7].

Theorem 7. Suppose that $\Gamma$ is rectifiable. Let $\Delta^{\mathrm{tp}} \supset\left\{\Omega_{n}\right\}_{n=1}^{\infty}$ be such that $\lim _{n \rightarrow \infty}\left|\Omega_{n}\right|=0$, and let $\left\{x_{n} \in X_{\Gamma, \Omega_{n}}^{\mathrm{tp}}\right\}_{n=1}^{\infty}$ be a sequence of the $d$-Douglas solutions.

Then there exists a subsequence $\left\{x_{n_{i}}\right\}$ which converges to one of the Douglas solutions $x \in X_{\Gamma}^{\mathrm{t} p}$ in the following sense:

$$
\lim _{n_{i} \rightarrow \infty}\left\|x-x_{n_{i}}\right\|_{H^{1}\left(D ; \mathbf{R}^{n}\right)}=0
$$

and if $x \in W^{1, p}\left(D ; \mathbf{R}^{n}\right), p>2$, then

$$
\lim _{n_{i} \rightarrow \infty}\left\|x-x_{n_{i}}\right\|_{C\left(\bar{D} ; \mathbf{R}^{n}\right)}=0 .
$$

If the Douglas solution is unique, then $x_{n}$ converges in the sense of (4.1) and (4.2).

A harmonic map $x \in X_{\Gamma}^{\mathrm{tp}}$ is said to be an isolated stable minimal surface if there exists a constant $\delta$ such that

$$
0<\|x-y\|_{C\left(\bar{D} ; \mathbf{R}^{n}\right)}<\delta \quad \text { implies } \quad E(x)<E(y) \text { for } y \in X_{\Gamma}^{\mathrm{tp}} .
$$

Theorem 8. Suppose that $\Gamma$ is rectifiable. Let $\Delta^{\mathrm{tp}} \supset\left\{\Omega_{n}\right\}_{n=1}^{\infty}$ be such that $\lim _{n \rightarrow \infty}\left|\Omega_{n}\right|=0$, and let $x \in X_{\Gamma}^{\mathrm{tp}}$ be an isolated stable minimal surface. Then there exists a sequence $\left\{x_{n} \in X_{\Gamma, \Omega_{n}}^{\mathrm{tp}}\right\}_{n=1}^{\infty}$ of stable $d$-minimal surfaces which converges to $x$ in the sense of (4.1) and (4.2).

\section{ACKNOWLEDGMENT}

The author would like to thank the referee for suggesting a number of improvements and corrections to the original version of this paper. 


\section{BIBLIOGRAPHY}

1. P. G. Ciarlet and P. A. Raviart, Maximum principle and uniform convergence for the finite element method, Comput. Methods Appl. Mech. Engrg. 2 (1973), 17-31.

2. R. Courant, Dirichlet's principle, conformal mapping, and minimal surfaces, Interscience, New York, 1950.

3. R. Courant and D. Hilbert, Methods of mathematical physics, Vol. II, Interscience, New York, 1962.

4. H. B. Lawson, Jr., Lectures on minimal submanifolds, Vol. I, Publish or Perish, Berkeley, 1980.

5. R. Osserman, A survey of minimal surfaces, 2nd ed., Dover, New York, 1986.

6. T. Tsuchiya, On two methods for approximating minimal surfaces in parametric form, Math. Comp. 46 (1986), 517-529.

7. _ Discrete solution of the Plateau problem and its convergence, Math. Comp. 49 (1987), 157-165.

8. W. L. Wilson, Jr., On discrete Dirichlet and Plateau problems, Numer. Math. 3 (1961), 359373.

Department of Mathematics, Kyushu University 33, Fukuoka, 812, Japan

Current address: Department of Mathematics, University of Maryland, College Park, Maryland 20742.E-mail: tak@wam.umd.edu 